\title{
Analytical Mechanics of Chemical Reactions. V. Application to the Linear Reactive $\mathrm{H}+\mathrm{H}_{2}$ Systems*
}

\author{
Sirtou-fu Wu and R. A. Marcus \\ Noyes Chemical Laboratory, Unizersity of Illinois, Urbana, Illinois 61801
}

(Received 8 July 1970)

\begin{abstract}
Natural collision coordinates and a zeroth-order vibrational-adiabatic approximation are used to treat linear reactive collisions. Nonadiabatic effects on barrier transmission and on vibrational state of products are calculated. The present results are classical and are compared with exact classical numerical results for the $\mathrm{H}+\mathrm{H}_{2}$ reaction in the range $7-20 \mathrm{kcal} / \mathrm{mol}$ of initial relative translational energy. The agreement is encouraging and the results support the concepts introduced earlier of statistical adiabaticity and of nonadiabatic leak. At low energies the reaction is adiabatic on the average (initial vibrational phase average), thus justifying activated complex theory for this system. The relative importance of reaction path curvature and of vibrational frequency variation along the reaction path in inducing nonadiabatic effects is described. Implications for a quantum treatment, activated complex theory, and highly nonadiabatic systems are noted.
\end{abstract}

\section{INTRODUCTION}

A formalism for the mechanics of nearly vibrationally adiabatic chemical reactions, $\mathrm{A}+\mathrm{BC} \rightarrow \mathrm{AB}+\mathrm{C}$, was described in earlier papers of this series. ${ }^{1,2}$ In the classical version $^{2}$ the Hamilton-Jacobi equation, expressed in terms of natural collision coordinates and action-angle variables, was used. A number of applications of these natural collision coordinates have recently appeared ${ }^{3-5}$ and include numerical (quantum and classical) and adiabatic treatments.

Action-angle variables, which were used to treat atomic and molecular structure many years ago, ${ }^{6}$ have been used more recently in this laboratory to treat inelastic and reactive collisions. ${ }^{2,7-9}$ Their use led to relatively simple approximate expressions for energy transfer and other properties. Encouraging agreement with exact results was obtained for the inelastic systems. ${ }^{7.8}$ The method provided information about alternative quantum approximations (near static vs near adiabatic), because of the close relationship which the HamiltonJacobi equation bears to the Schrödinger equation and which the action variables $J_{i}$ bear to the quantum numbers $n_{i}\left(J_{i} / h \cong n_{i}+\frac{1}{2}\right.$ or $n_{i}$, depending upon coordinate). The approximations are particularly suited to near-elastic or near-adiabatic systems.

The rearrangement reaction $\mathrm{H}+\mathrm{H}_{2} \rightarrow \mathrm{H}_{2}+\mathrm{H}$ has been intensively studied both classically $y^{10-13}$ and quantum mechanically, ${ }^{\text {,a }, 5,14-16}$ largely though not exclusively numerically. The present paper calculates and compares, for the first time, nonadiabatic corrections with exact numerical results. Some quantum mechanical implications are noted in a concluding section. Results on reaction in a plane will be given in a subsequent paper.

\section{EXACT CLASSICAL EQUATIONS WITH NATURAL COLLISION COORDINATES}

As in Paper IV, the reaction coordinate and the vibrational coordinate are denoted by $s$ and $\rho$, respectively; a suitable curve $C$ is drawn in a mass-weighted configuration space (in the center-of-mass system), $s$ being the arc length along this curve (e.g., Fig. 1).
The Cartesian coordinates of the space are $z$ and $Z$. The curvature of curve $C, \kappa(s)$, is a function of $s$. The reaction corresponds to a suitable motion of a point of mass $\mu$ from region I to region II in Fig. 1. Hamilton's equations of motion in natural collision coordinates, $s$ and $\rho$, are obtained from Eqs. (3) and (4) of Paper IV in the standard way, ${ }^{17}$

$$
\begin{aligned}
\dot{p} & =\frac{\rho(d \kappa / d s) p_{s}^{2}}{\mu \eta^{3}}-\frac{\partial V}{\partial s}, \\
\dot{s} & =p_{s} / \mu \eta^{2} \\
\dot{p}_{\rho} & =\left(\kappa p_{s}{ }^{2} / \mu \eta^{3}\right)-(\partial V / \partial \rho), \\
\dot{\rho} & =p_{\rho} / \mu
\end{aligned}
$$

where the $p$ 's denote momenta conjugate to $s$ and $\rho$ and where

$$
\eta=1+\kappa \rho .
$$

The force components in Eqs. (1) along the natural collision coordinates, $-\partial V / \partial \rho$ and $-\partial V / \partial s$, are obtained from the interatomic forces using the "chain rule,"

$$
\frac{\partial V}{\partial q^{i}}=\sum_{k=1}^{3} \sum_{l=1}^{2} \frac{\partial V}{\partial R_{k}} \frac{\partial R_{k}}{\partial Q^{l}} \frac{\partial Q^{l}}{\partial q^{i}}
$$

where $q^{i}=s, \rho, Q^{l}=z, Z$, and $R_{1}, R_{2}$, and $R_{3}$ are the internuclear distances. The four simultaneous differential equations (1) can be integrated numerically.

\section{APPROXIMATE EQUATIONS FOR THE VIBRATIONAL $\rho$-MOTION}

The potential energy function $V(\rho, s)$ is written ${ }^{2}$ in terms of its value on curve $C, V_{1}(s)$, and the increment to a finite $\rho$ at this $s, V_{2}(\rho, s)$,

$$
V(\rho, s)=V_{1}(s)+V_{2}(\rho, s) .
$$

The motion along the reaction coordinate creates a mean "internal centrifugal potential" acting on the $\rho$ motion a potential arising from the $\kappa p_{s}{ }^{2} / \mu \eta^{3}$ term in (1c) and denoted by $\alpha(s) / \eta^{2}$, with $\alpha(s)$ defined below. It is sometimes useful, but not necessary, to incorporate 
the $\alpha / \eta^{2}$ term in the zeroth-order treatment of the $\rho$ motion, and we do this in the present case":

The effective potential $U(\rho, s)$ for the $\rho$ motion when $\alpha / \eta^{2}$ is included is ${ }^{2}$

$$
U(\rho, s)=V_{2}(\rho, s)+\left(\alpha / \eta^{2}\right),
$$

where $\alpha(s)$ denotes the average $p_{s}^{2} / 2 \mu$ in the adiabatic approximation ${ }^{18}$ :

$$
\alpha(s) / \eta_{0}^{2}=E-E_{\mathrm{rib}^{\mathrm{ad}}}(s)-V_{1}(s)-V_{2}\left(\rho_{\mathrm{v}}, s\right),
$$

where

$$
\eta_{0}=1+\kappa \rho_{0} .
$$

The $\rho_{0}(s)$ and the adiabatic vibrational energy $\left[E_{\mathrm{vib}^{\mathrm{ad}}}{ }^{\mathrm{ad}}(s)\right]$ in (6) are given below. $\alpha(s)$ is found by solving $(6),(7)$, and (9) iteratively or simultaneously. [An iteration can be initiated by setting $V_{2}\left(\rho_{0}, s\right)=0$ in (6) and $\partial^{2} U / \partial \rho^{2}=\partial^{2} V / \partial \rho^{2}$ in (9) in the first step.]

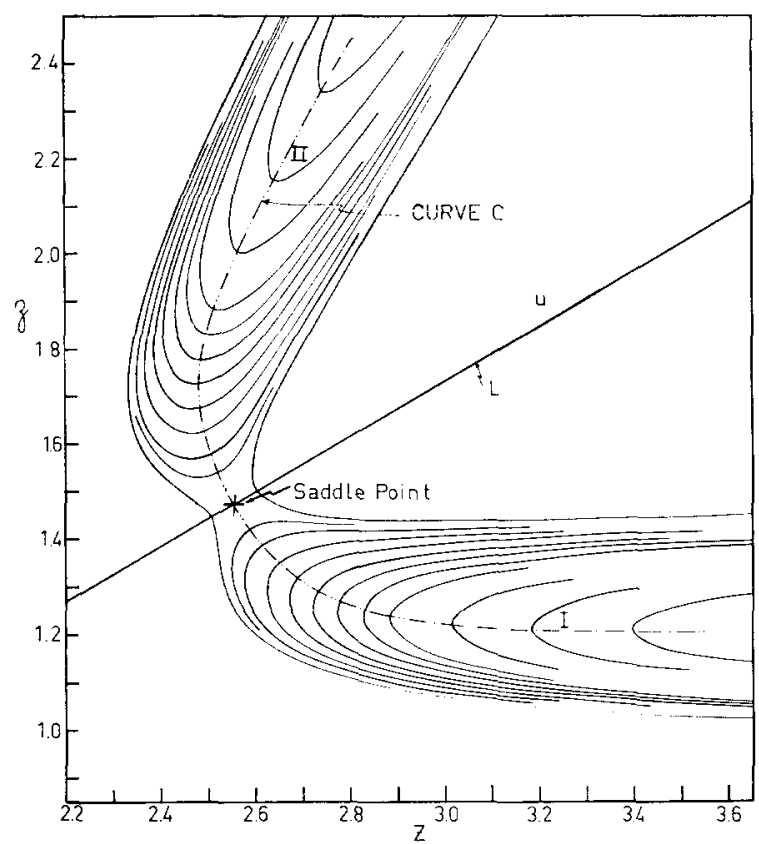

FIG. 1. Potential energy contour map for $\mathrm{H}_{3}$ (relative to $\mathrm{H}, \mathrm{H}_{2}$ as zero) in the $(z, Z)$ plane for linear configurations. Curve $C$ $(-\cdots)$ is chosen graphically as the curve which is steepest descent from the saddle point down to the reactant and product valleys, respectively.

The value of $\rho$ which minimizes $U(\rho, s)$ at any $s$ is denoted $^{2}$ by $\rho_{0}(s)$,

$$
\partial U(\rho, s) / \partial \rho=0 \text { at } \rho=\rho_{0}(s) .
$$

The vibrational variables $\rho$ and $p_{\rho}$ can be expressed, at any $s$, in terms of local action-angle variables for the $\rho$ motion, $J_{\rho}$ and $w_{\rho}$, respectively. ${ }^{2}$ In physical systems the initial value of $w_{\rho}$ is random in the interval $(0,1)$. In the adiabatic approximation $J_{\rho}$ is a constant of the motion. When the $\rho$ motion is treated as locally harmonic, we have ${ }^{2}$

$$
U(\rho, s) \cong U\left(\rho_{0}, s\right)+\frac{1}{2} \mu \omega^{2}\left(\rho-\rho_{0}\right)^{2},
$$

Table I. The points chosen for least-square fit of the reaction path (i.e., Curve $C$ ) and their potentials along the reaction path.

\begin{tabular}{lll}
\hline \hline z (a.u.) & $Z$ (a.u.) & $V_{1}(s)$ (a.u.) \\
\hline 1.4731 & 2.5515 & 0.014563 \\
1.4229 & 2.5855 & 0.014344 \\
1.3908 & 2.6103 & 0.013975 \\
1.3484 & 2.6500 & 0.013240 \\
1.3150 & 2.6856 & 0.012505 \\
1.2951 & 2.7128 & 0.012000 \\
1.2870 & 1.7263 & 0.011767 \\
1.2668 & 2.7736 & 0.011033 \\
1.2554 & 2.8035 & 0.010600 \\
1.2524 & 2.8266 & 0.010298 \\
1.2419 & 2.8849 & 0.009562 \\
1.2342 & 2.9351 & 0.008975 \\
1.2261 & 3.0186 & 0.008091 \\
1.2214 & 3.1096 & 0.007239 \\
1.2191 & 3.1838 & 0.006620 \\
1.2140 & 3.3988 & 0.005147 \\
1.2138 & 3.5911 & 0.004149 \\
1.2136 & 3.8490 & 0.003151 \\
1.2134 & 4.2314 & 0.002150 \\
\hline
\end{tabular}

where

$$
\mu \omega^{2}(s)=\partial^{2} U(\rho, s) / \partial \rho^{2} \quad \text { at } \rho=\rho_{0}(s) .
$$

$E_{\text {vib }}$ has a very simple form for a locally harmonic oscillator, ${ }^{2}$

$$
E_{\mathrm{vib}}=J_{\rho} \omega(s) / 2 \pi \text {. }
$$

The adiabatic value of $E_{\mathrm{vib}}$ is obtained for a reaction by setting $J_{\rho}$ equal to its initial value, $J_{\rho}^{0}$,

$$
E_{\mathrm{vib}}^{\text {ad }}(s)=J_{\rho}{ }^{0} \omega(s) / 2 \pi \text {. }
$$

In the local harmonic approximation, which we now adopt in the present section, the equations of motion for $J_{\rho}$ and $w_{\rho}$ are ${ }^{2}$

$$
\begin{array}{r}
\dot{J}_{\rho}=-2 \dot{\rho}_{0}\left(\mu \omega \pi J_{\rho}\right)^{1 / 2} \sin 2 \pi w_{\rho}-\left(J_{\rho} \dot{\omega} / \omega\right) \cos 4 \pi w_{\rho}, \\
\dot{w}_{\rho}=(\omega / 2 \pi)-\frac{1}{2} \dot{\rho}_{v}\left(\mu \omega / J_{\rho} \pi\right)^{1 / 2} \cos 2 \pi w_{\rho} \\
+(\dot{\omega} / 4 \pi \omega) \sin 4 \pi \omega_{\rho} .
\end{array}
$$

To remove the singularity in the differential equations (11) and (12) at $J_{\rho}=0$, a transformation of

TABLE II. The least-square fitted coefficients of the reaction path (i.e., Curve $C$ ).

\begin{tabular}{ccc}
\hline & & $-0.50 \geq v \geq-0.90 \mathrm{a} . \mathrm{u}$. \\
& $0.5 \geq v \geq-0.50 \mathrm{a} . \mathrm{u}$. & $\begin{array}{c}0.50 \leq v \leq 0.90 \\
\text { and } 0.50\end{array}$ \\
$A_{0}$ & 2.9469511 & 2.8374358 \\
$A_{1}$ & 0.81743033 & 2.2616786 \\
$A_{2}$ & 4.5372891 & -1.8003946 \\
$A_{3}$ & -8.5215932 & 1.2827108 \\
$A_{4}$ & -4.0067625 & -0.5147454 \\
$A_{5}$ & 16.367035 & 0.08376852 \\
\hline \hline
\end{tabular}




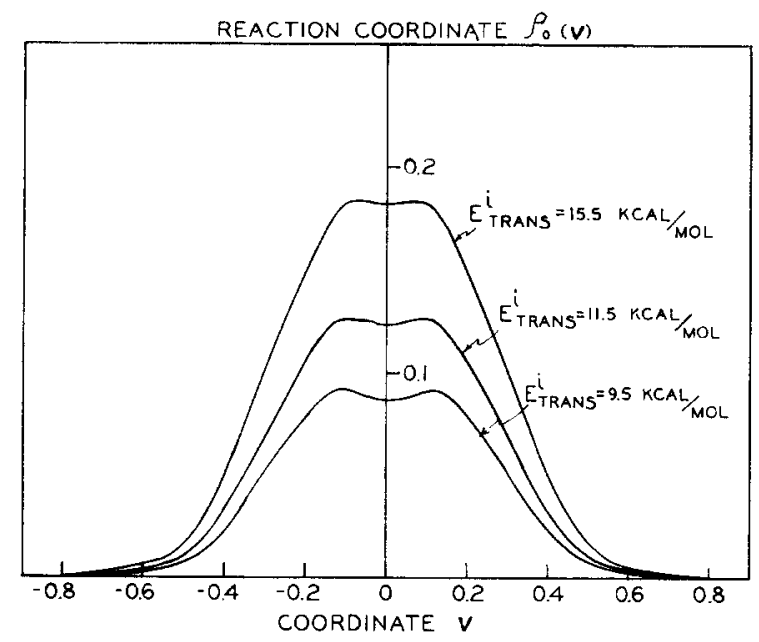

FIG. 2. Reaction coordinate $\rho_{0}(v)$ as a function of coordinate 0 for three initial relative translational energies, $E_{\operatorname{trans}}{ }^{i}=9.5$, 11.5 , and $15.5 \mathrm{kcal} / \mathrm{mol}$.

variables from $\left(J_{\rho}, w_{\rho}\right)$ to canonically conjugate variables $a$ and $a^{*}$ can be performed, ${ }^{2}$ where $a$ is $J_{\rho}{ }^{1 / 2} \exp \left(-2 \pi i w_{\rho}\right)$, yielding ${ }^{2}$

$$
\dot{a}=-i \omega a+i \dot{\rho}_{0}(\mu \omega \pi)^{1 / 2}-\left(\dot{\omega} a^{*} / 2 \omega\right) .
$$

$a^{*}$ is the complex conjugate of $a$.

When the $\dot{\omega}$ term in (13) is treated as a perturbation, integration of (13) yields (cf. Eqs. 93 and 94 of Paper IV) ${ }^{19}$

$$
\begin{aligned}
& J_{p}=J_{\rho}{ }^{0}-2\left(J_{\rho}{ }^{0}\right)^{1 / 2} \int_{-\infty}^{t} {\left[\dot{\rho}_{0}(\mu \omega \pi)^{1 / 2}\right.} \\
&\left.\times \sin \left(\int_{0}^{t^{\prime}} \omega d t^{\prime \prime}+\delta_{\rho}\right)\right] d t^{\prime}+\mid \int_{-\infty}^{t}\left[\dot{\rho}_{0}(\mu \omega \pi)^{1 / 2}\right. \\
&\left.\quad \times \exp \left(i \int_{t^{\prime}}^{t} \omega d t^{\prime \prime}\right)\right]\left.d t^{\prime}\right|^{2}+\Delta
\end{aligned}
$$

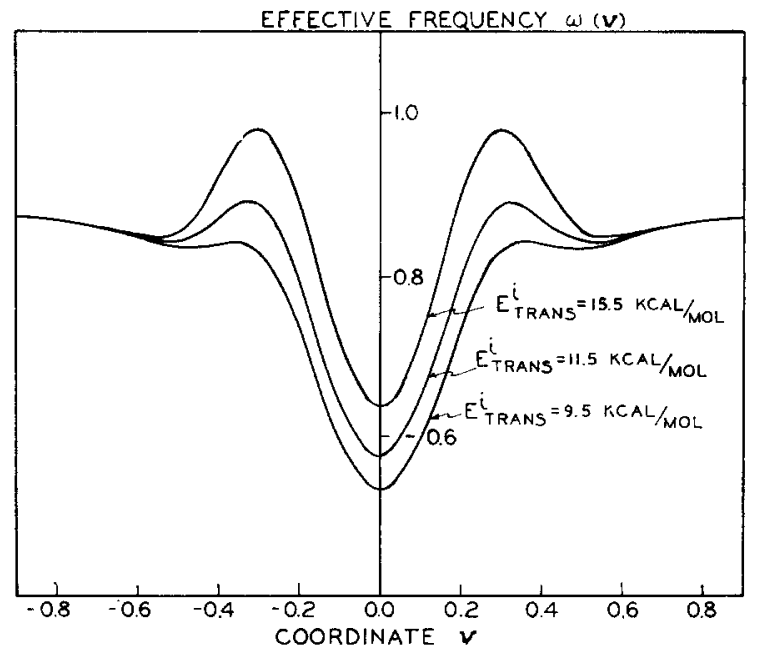

FIG. 3. Effective frequency $\omega(v)$ of the vibrational $\rho$ motion as a function of coordinate $v$ for three initial relative translational energies, $E_{\text {trans }}{ }^{i}=9.5,11.5$, and $15.5 \mathrm{kcal} / \mathrm{mol}$. where $\Delta$ is the perturbation due to the $\dot{\omega}$ term:

$$
\Delta=-J_{p}^{0} \int_{-\infty}^{t}\left[\frac{\dot{\omega}}{\omega} \cos 2\left(\int_{0}^{t^{\prime}} \omega d t^{\prime \prime}+\delta_{\rho}\right)\right] d t^{\prime} .
$$

When both the $\dot{\rho}_{0}$ and $\dot{\omega}$ terms in (13) are treated as perturbations to first order, Eq. (14) is again obtained, ${ }^{2}$ apart from the $\delta_{\rho}$.independent term (the third term) on the right-hand side of (14). In effect, therefore, Eq. (14) treats the $\dot{\omega}$ term to first order and the $\dot{\rho}_{0}$ term to second or higher order.

\section{APPLICATION TO THE $\mathbf{H}+\mathrm{H}_{2} \rightarrow \mathrm{H}_{2}+\mathrm{H}$ REACTION}

\section{A. Potential Energy Surface, Curve $C$, and Other Properties}

A number of studies have been made on obtaining the best potential energy surface for $\mathrm{H}_{3}$ system. ${ }^{20} \mathrm{We}$ employ the Porter and Karplus surface, ${ }^{21}$ previously used in extensive trajectory ${ }^{11}$ and other scattering calculations. ${ }^{16}$ The contour of the potential energy surface expressed in the skewed-coordinates is plotted in Fig. 1.

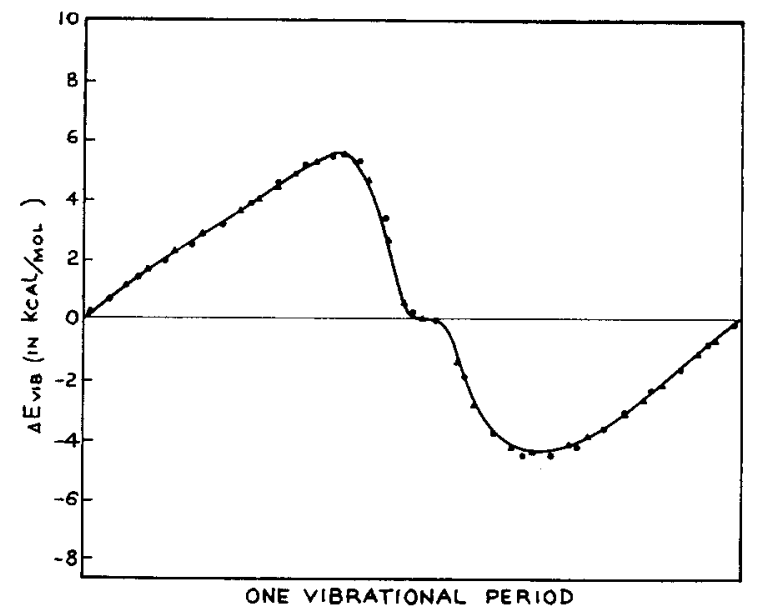

FIG. 4. Comparison of exact calculations for $\Delta E_{\mathrm{vib}}$ 's based on natural collision coordinates $(\bullet)$ and on ordinary coordinates $(\boldsymbol{A})$; linear collision of $\mathrm{H}+\mathrm{H}_{2}$ system having $E_{\text {trans }}{ }^{i}=9.5 \mathrm{kcal} / \mathrm{mol}$.

A curve $C$ can be obtained from the contours, for example, by drawing the curve of the steepest descent from the saddle point. For numerical purposes this curve (the points of which are given in Table I) was fitted to a polynomial by least-square method. If the $(z, Z)$ axes of Fig. 1 are rotated by $30^{\circ}$ about the saddle-point into $(u, v)$ axes to utilize the symmetry of the surface, the polynomial equation describing curve $C$ is, in these $(u, v)$ coordinates,

$$
\begin{array}{ll}
u=-(3 v)^{1 / 2}+2 r_{0} & \text { for } \quad v<-0.9 \text { a.u., } \\
u=(3 v)^{1 / 2}+2 r_{0} & \text { for } \quad v>0.9 \text { a.u., } \\
u=\sum_{l=0}^{6} A v^{2 l} & \text { for } \quad-0.9 \leq v \leq 0.9 \text { a.u. }
\end{array}
$$


where $r_{0}$ is the equilibrium bond length (in reduced, mass-weighted units) and where the coefficients $A_{l}$ are given in Table II. The saddle-point occurs at $u=0, v=0$.

Using this curve $C, \rho_{0}(s)$ and $\omega(s)$ were calculated from Eqs. (7) and (9) for various energies, and are shown for several energies in Figs. 2 and 3. As seen from these plots both quantities have already effectively approached their asymptotic values at $|v|=0.9$ a.u. Thus, it is a good approximation to truncate the calculation at this $|v|$.

Both $\rho_{0}(s)$ and $\omega(s)$ were fitted to a polynomial in $v$ by the least-square method to obtain an analytical expression for these quantities.

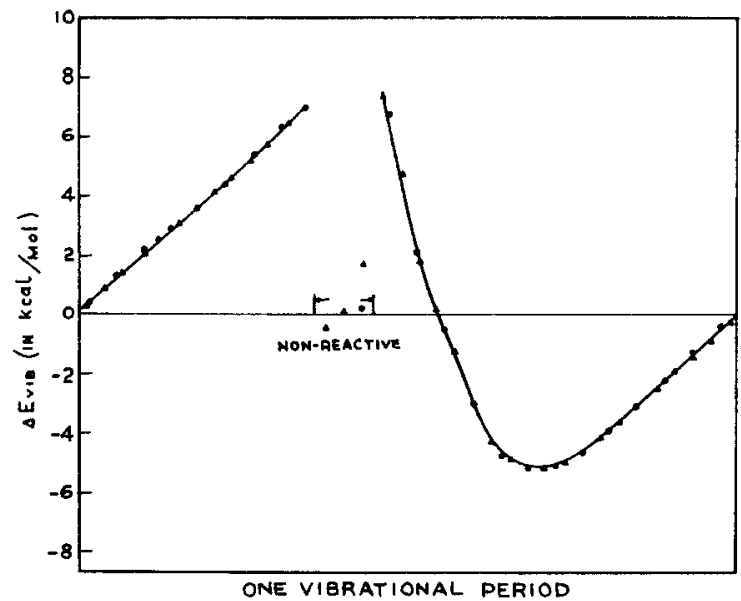

FIG. 5. Comparison of exact claculations for $\Delta E_{\mathrm{vib}}$ 's based on natural collision coordinates $(\bullet)$ and on ordinary coordinates $(\boldsymbol{A})$; linear collision of $\mathrm{H}+\mathrm{H}_{2}$ system having $E_{\mathrm{tran}}{ }^{i}=10.55 \mathrm{kcal} / \mathrm{mol}$ ).

\section{B. Comparison of Exact Calculations Based on Eqs. (1) with Those Based on Cartesian Coordinates, $z$ and $Z$}

Before comparing the results based on Eqs. (14) with those based on exact (numerical) integration in ordinary center-of-mass coordinates, it is useful to compare the results of the latter with those based on the exact integration of (1). The results of these purely numerical integrations are compared as a function of initial vibrational phase in Figs. 4 and 5. To obtain these figures the equations of motion in each set of coordinates [Eq. (1) for natural collision coordinates and the usual equations for ordinary coordinates $\left.{ }^{22}\right]$ were integrated by a fourth-order Runge-Kutta-Gill method with the appropriate boundary conditions. ${ }^{23} \mathrm{~A}$ total number of 36 vibrational phases were calculated for each initial relative translational energy. The agreement for the reactive collisions is seen from Figs. 4 and 5 to be excellent.

Examination of Eqs. (1) and (2) shows that the use of natural collision coordinates introduces a singularity at $\kappa \rho=-1$, and indeed an ambiguity in the coordinate definition in this region. ${ }^{24}$ If a trajectory approached

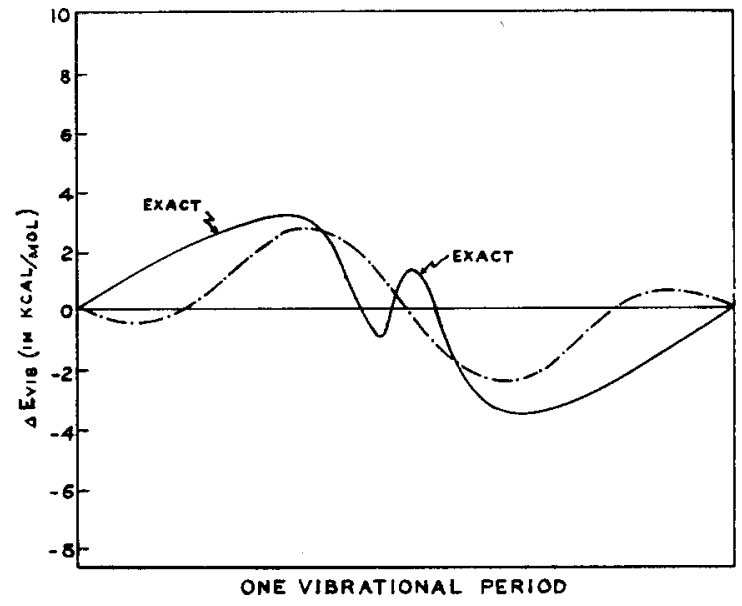

Fig. 6. Vibrational-translation energy transfer in a linear collision, as a function of initial vibrational phase: - exact classical calculation; ---, approximate calculation, for $E_{\mathrm{trans}}{ }^{i}=$ $8.5 \mathrm{kcal} / \mathrm{mol}$.

this singularity or reached points corresponding to negative values of $1+\kappa \rho$, i.e., points on the other side of the singularity from curve $C$ in Fig. 1, an error would result from the use of these natural collision coordinates. The excellent agreement of the two calculations for the reactive collisions shows that the singularity was not approached closely (at least for the cases treated in those figures). Figure 4 gives perhaps an indication of some difference for the nonreactive case, and indeed, as an examination of nonreactive trajectories will show later, that the system came closer to the singularity in this case.

\section{Approximate Results Based on Eq. (14)}

The adiabatic solution for $s(t)$ satisfies ${ }^{2}$

$$
\dot{s}=[2 \alpha(s) / \mu]^{1 / 2}\left(1+\kappa \rho_{0}\right)^{-2},
$$

where $\alpha(s)$ is given by (6). Instead of $s(t)$ the co-

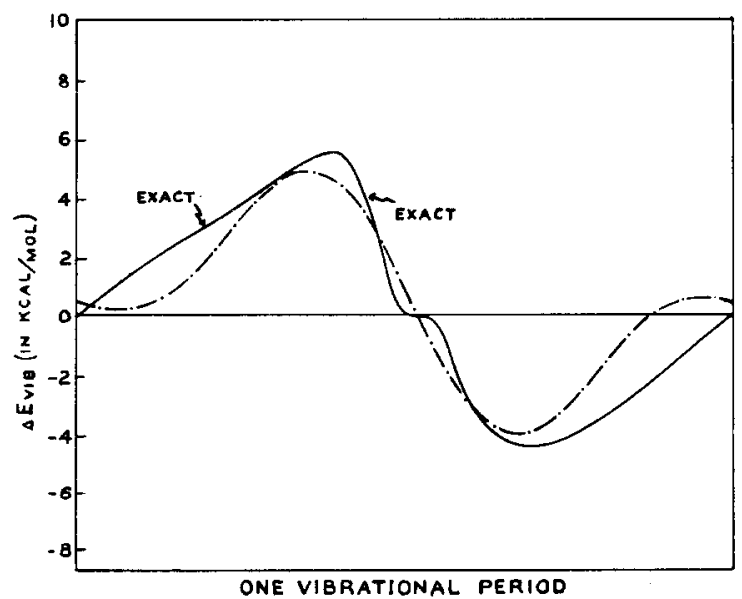

FIg. 7. Vibrational-translational energy transfer in a linear collision, as a function of initial vibrational phase: - exact classical calculation; -- - , approximate calculation, for $E_{\text {trans }}{ }^{i}=9.5$ $\mathrm{kcal} / \mathrm{mol}$. 


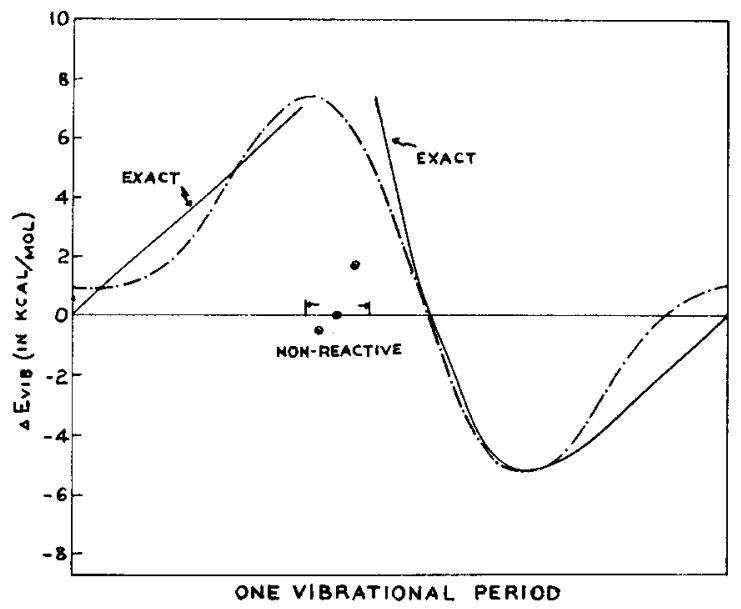

FIG. 8. Vibrational-translational energy transfer in a linear collision, as a function of initial vibrational phase: - , exact classical calculation; ---, approximate calculation, for $E_{\operatorname{tran}}{ }^{i}=$ $10.55 \mathrm{kcal} / \mathrm{mol}$. The circled points refer to nonreactive collisions.

ordinate $v(t)$ defined earlier can be used, in which case (17) would become

$$
\dot{v}=[2 \alpha(s) / \mu]^{1 / 2}\left[1+(d u / d v)^{2}\right]^{-1 / 2}\left(1+\kappa \rho_{0}\right)^{-2} .
$$

While there was little advantage in using $v(t)$ instead of $s(t)$, provided the line $s=0$ was chosen to lie along the symmetry axis $L$ of Fig. 1, i.e., at $v=0,(18)$ was used instead of (17).

The phase $\delta_{\rho}$ in (14) is the vibrational phase when the system crosses the symmetry axis $L$ in Fig. 1, i.e., the activated complex line. The trigonometric terms in (14) and (15) can be rewritten using the addition formulas, so that the phase $\delta_{\rho}$ appears outside each integral. Integrals denoted by $S_{1}, C_{1}, S_{2}$, and $C_{2}$ may be defined:

$$
\begin{gathered}
S_{1}=\int_{-\tau_{0}}^{0}(\mu \omega \pi)^{1 / 2} \sin 2 \pi w_{\rho}^{\prime} d t, \\
C_{1}=\int_{-\tau_{0}}^{0}(\mu \omega \pi)^{1 / 2} \cos 2 \pi w_{\rho}^{\prime} d t, \\
S_{2}=\int_{-\tau_{0}}^{0} \frac{\dot{\omega}}{\omega} \sin 4 \pi w_{\rho}^{\prime} d t, \\
C_{2}=\int_{-\tau_{0}}^{0} \frac{\dot{\omega}}{\omega} \cos 4 \pi \omega_{\rho}^{\prime} d t,
\end{gathered}
$$

where $-\tau_{0}$ is the initial time, i.e., the time when $v=-v_{\mathrm{i}}$, and where the $w_{\rho}^{\prime}$ in (19) denotes

$$
2 \pi w_{\rho}^{\prime}=\int_{0}^{t} \omega d t
$$

$J_{\rho}$ at the (truncated) final time $\left(t=\tau_{0}\right)$ is found from (14) to be

$J_{\rho}\left(t=\tau_{0}\right)=J_{\rho}^{0}-4\left(J_{\rho}^{0}\right)^{1 / 2} S_{1} \cos \delta_{\rho}+4 S_{1}{ }^{2}+2 J_{\rho}{ }^{0} C_{2} \sin 2 \delta_{\rho}$.
$J_{\rho}$ at the saddle point, $J_{\rho}(l=0)$, is cimilarly found to be

$$
\begin{aligned}
J_{\rho}(t=0)= & J_{\rho}{ }^{0}-2\left(J_{\rho}^{0}\right)^{1 / 2}\left(S_{1} \cos \delta_{\rho}+C_{1} \sin \delta_{\rho}\right) \\
& +S_{1}{ }^{2}+C_{1}{ }^{2}+\left(C_{2} \sin 2 \delta_{\rho}-S_{2} \cos 2 \delta_{\rho}\right) J_{\rho}{ }^{0} .
\end{aligned}
$$

The $\delta_{p}{ }^{0}$ used to represent the approximate results in Figs. 6-11 is related to $\delta_{\rho}$ by

$$
\hat{o}_{\rho}{ }^{\prime \prime}=\delta_{\rho}-\int_{-\tau_{0}}^{0} \omega d t
$$

The integrals in $\left(19^{\prime}\right)$ and (22) were computed using a six-point Gauss-Legendre quadrature for an integration inverval, $\Delta v=0.005$ a.u. The integrals $S_{1}, C_{1}, S_{2}$, and $C_{2}$ in (19) were next calculated by Simpson's rule. The value of $J_{\rho}$ was then obtained from (20) and (21) at the product and saddle-point regions, respectively. The integrals are tabulated in Table III.

The change of vibrational energy $\Delta E_{\mathrm{vib}}$ was calculated both from the exact Hamilton's equations of motion and from the above equations for a series of initial relative translational energies, $E_{\text {trans }}{ }^{i}=8.5-15.5$ $\mathrm{kcal} / \mathrm{mol}$, and initial vibrational phases. ${ }^{25}$ The results are given in Figs. 6-11.

Equations (6) and (17) yield the adiabatic threshold for reaction. However, for certain $\delta_{\rho}$, the actual vibrational energy $E_{\mathrm{vib}}\left(s, \delta_{p}\right)$ may exceed $E_{\mathrm{vib}}(s)$ and so cause reflection at energies above the adiabatic threshold. Reflection occurs when the actual $\dot{s}$ vanishes, namely, when

$$
E-E_{\mathrm{vib}}\left(s, \delta_{\rho}\right)-V_{1}(s)-V_{2}\left(\rho_{\mathrm{c}}, s\right) \leq 0
$$

at some $s$. Equation (23) can be solved for $\delta_{\rho}$ and for $s$, which will be near $s=0$ since $V_{1}(s)$ has a maximum there. If $s=0$ is used in $(23),{ }^{26}$ and the latter is solved for $\delta_{\rho}$, the nonreactive collisions so defined are indicated by vertical lines on the abscissas in Figs. 6-11. The range of nonreactive phase is seen to increase, and therefore, the reaction probability decreases, with increasing initial relative translational energy, at the higher energies, in the energy range considered.

Comparing the reactive portion in Figs. 6-11, we see that there is good agreement between the exact and

TABLE III. Values of integrals, $S_{1}, C_{1}, S_{2}$, and $C_{2}$.

\begin{tabular}{ccccc}
\hline \hline $\begin{array}{c}E_{\text {trans }}{ }^{i} \\
(\mathrm{kcal} / \mathrm{mol})\end{array}$ & $S_{1}$ & $C_{1}$ & $S_{2}$ & $C_{2}$ \\
\hline 7.5 & 0.005330 & -0.037280 & 0.080769 & 0.088301 \\
8.5 & -0.014951 & -0.049132 & 0.093976 & 0.126385 \\
9.5 & -0.033779 & -0.056788 & 0.103261 & 0.164801 \\
10.55 & -0.052518 & -0.062774 & 0.112568 & 0.204917 \\
11.5 & -0.068083 & -0.068069 & 0.112378 & 0.229329 \\
12.5 & -0.084476 & -0.072271 & 0.115724 & 0.255077 \\
13.5 & -0.099598 & -0.076646 & 0.117835 & 0.283105 \\
14.5 & -0.114736 & -0.079938 & 0.121419 & 0.303617 \\
15.5 & -0.128878 & -0.083120 & 0.121589 & 0.327182 \\
16.5 & -0.142668 & -0.086128 & 0.125010 & 0.347025 \\
17.5 & -0.156488 & -0.088406 & 0.120778 & 0.370785 \\
19.5 & -0.180849 & -0.093940 & 0.128212 & 0.400339 \\
\hline \hline
\end{tabular}


approximate calculations of the phase-dependent $\Delta E_{\mathrm{vib}}$ for most of the reactive regions. (In some instances the approximate curve sinusoidally winds around that of exact calculations in these figures, due to the character of the approximate Fourier series expansion employed.)

The phase-averaged vibrational energy change $\left\langle\Delta E_{\mathrm{vib}}\right\rangle$ accompanying the reaction is plotted for a series of $E_{\text {trans }^{i}}{ }^{i}$ in Fig. 12. The vibrational phaseaveraging procedure for the approximate calculation is merely the phase average over $\cos \delta_{\rho}$ and $\sin \delta_{\rho}$ in Eqs. (20) and (21). The average is over the range of reactive $\delta_{\rho}$ 's, with $\delta_{\rho}$ being random in this region. In Fig. 12 it is seen that the agreement between exact and approximate calculations of $\left\langle\Delta E_{\mathrm{vib}}\right\rangle$ is good on an absolute basis.

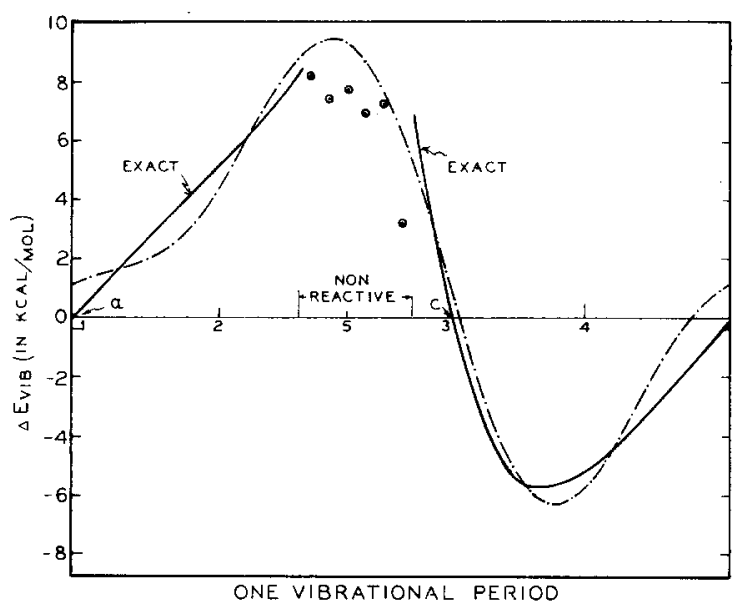

FIG. 9. Vibrational-translational energy transfer in a linear collision, as a function of initial vibrational phase: - , exact classical calculation; -.-, approximate calculation, for $E_{\mathrm{trans}}{ }^{i}=$ $11.5 \mathrm{kcal} / \mathrm{mol}$. The circled points refer to nonreactive collisions.

$\left\langle\Delta E_{\mathrm{vib}}\right\rangle$ is seen in Fig. 12 to be small at initial relative translational energies appropriate to the usual reaction temperatures of $500-1200^{\circ} \mathrm{K}$. (It can be shown that this range corresponds to $E_{\text {trans }}{ }^{i}$ in the neighborhood of $8.2-10.3 \mathrm{kcal} / \mathrm{mol}$.) Implications for activated complex theory are noted later.

The maximum $\dot{\rho}_{0}$ contribution and the maximum $\dot{\omega}$ contribution to (14), obtained by maximizing each contribution with respect to $\delta_{\rho}$, are plotted in Figs. 13 and 14 . The $\dot{\rho}$ term and the $\dot{\omega}$ term are roughly equally effective in introducing nonadiabatic transitions at the lower energies. At the higher energies, the $\dot{\rho}$ term is larger than the $\dot{\omega}$ term (and the same remark applies to the phase-dependent $\dot{\rho}$ term alone). This behavior supports the treatment which $\operatorname{led}^{2}$ to (14), since at low energies both terms need be treated only to first order while at higher energies the larger $\left(\dot{\rho}_{0}\right)$ contribution should be treated to higher order.

The reaction probability for the exact classical calculation is

$$
\text { Reaction Probability }=\left(\Delta \delta_{\rho}^{0}\right)_{\text {reactive }} / 2 \pi \text {, }
$$

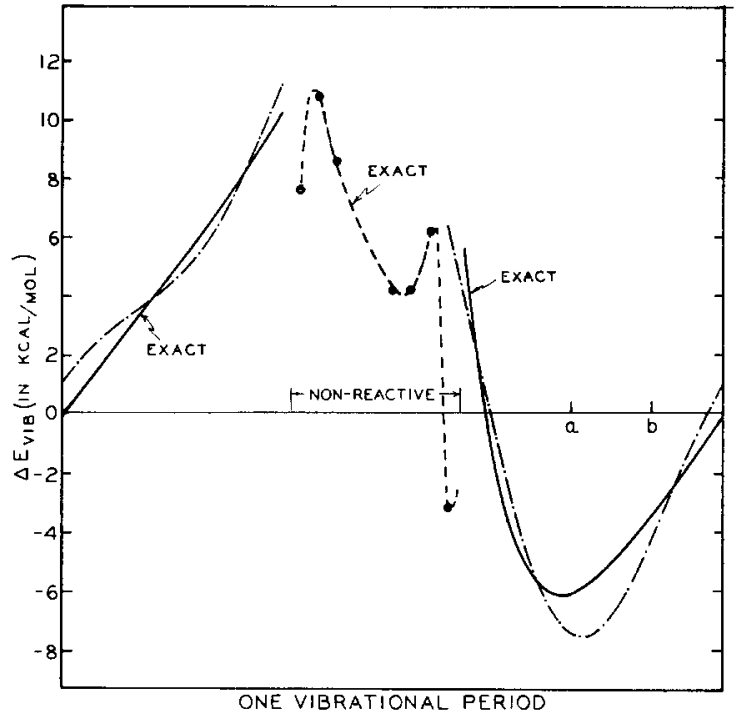

FIG. 10. Vibrational-translational energy transfer in a linear collision, as a function of initial vibrational phase: - exact classical calculation; -.-, approximate calculation, for $E_{\text {trans }} i=$ $13.5 \mathrm{kcal} / \mathrm{mol}$. The circled points and dashed line refer to nonreactive collisions.

where $\left(\Delta \delta_{p}^{0}\right)_{\text {reactive }}$ is the sum of intervals of initial vibrational phases for which the classical trajectories are reactive. The reaction probability is plotted versus initial translational energy in Fig. 15 and compared with one obtained using the approximate equations and the condition (23) at $s=0 .{ }^{27}$ The reaction probability is seen in Fig. 15 to rise rapidly to unity at energies above threshold and to provide a transmission at energies below the adiabatic threshold, i.e., to provide a "non-

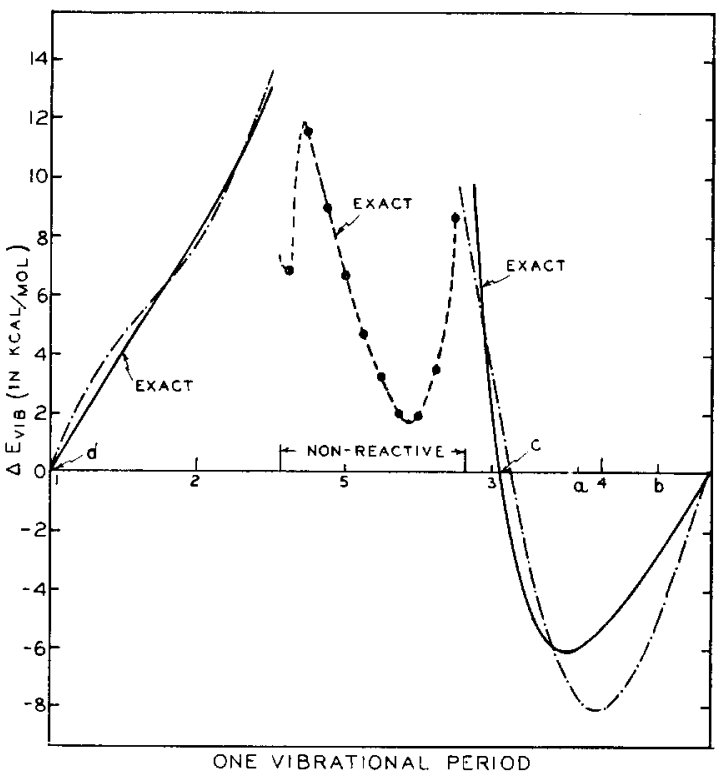

FIG. 11. Vibrational-translational energy transfer in a linear collision, as a function of initial vibrational phase: - , exact classical calculation; ---, approximate calculation, for $\vec{E}_{\mathrm{trans}}{ }^{i}=$ $15.5 \mathrm{kcal} / \mathrm{mol}$. The circled points and dashed line refer to nonreactive collisions. 


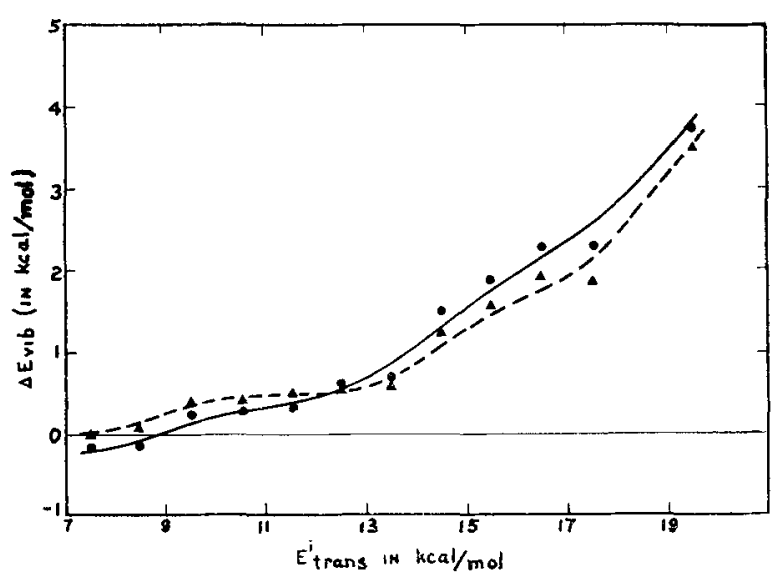

FIG. 12. Phase average of the vibrational-translational energy transfer for linear collision, as a function of initial relative translational energy: - -, exact; - $\mathbf{\Delta - - ;}$ approximate.

adiabatic leak." This same leak is responsible for the negative values of exact $\left\langle\Delta E_{\mathrm{vib}}\right\rangle$ at low initial translational energies in Fig. 12.

The vibrational action computed from Eq. (14) ${ }^{28}$ is given as a function of the coordinate $s$ in Figs. 16 and 17 for two initial translational energies and various vibrational phases. Several not unexpected points emerge in these figures: (1) The rate of change of $J_{\rho}$ along $s$ is least at large $|s|$, where there is no interaction, and at small $s$, i.e., in the vicinity of the saddle point, where the velocity $\dot{s}$ along the reaction coordinate is least. (2) The region where $E-E_{\mathrm{vib}}\left(s, \delta_{\rho}\right)-$ $V_{1}(s)-V_{2}\left(\rho_{0}, s\right)$ becomes negative probably does not necessarily occur at $s=0$, although $V_{1}(s)$ is a maximum there, since $E_{\mathrm{vib}}\left(s, \delta_{\rho}\right)\left[=J_{\rho}(s) \omega(s) / 2 \pi\right]$ sometimes has a local minimum at or near $s=0$. (3) Because of the increase in $J_{\rho}(s) \omega(s)$ after passage through $s=0$ in

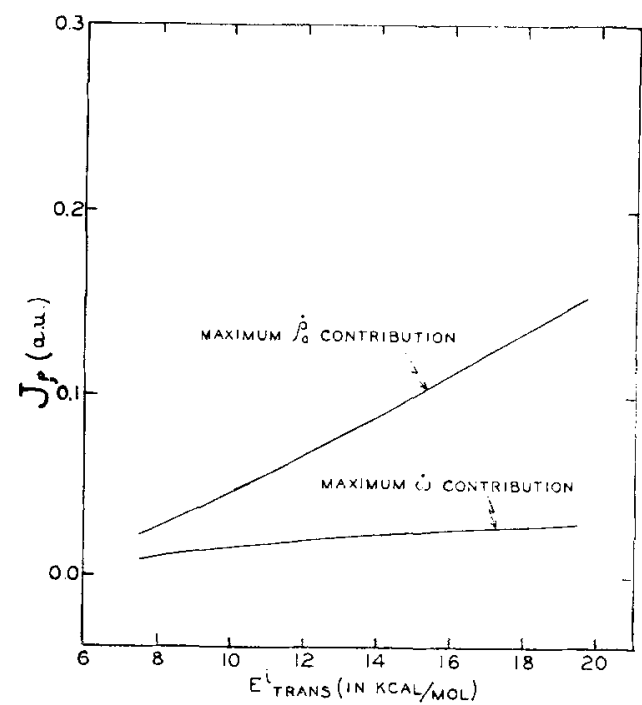

FIG. 13. Maximum contributions from $\dot{\rho}_{0}$ and $\dot{\omega}$ terms to the action $J_{\rho}$ at the saddle-point region (maximum over-all vibrational phases) as a function of initial relative translational energies.

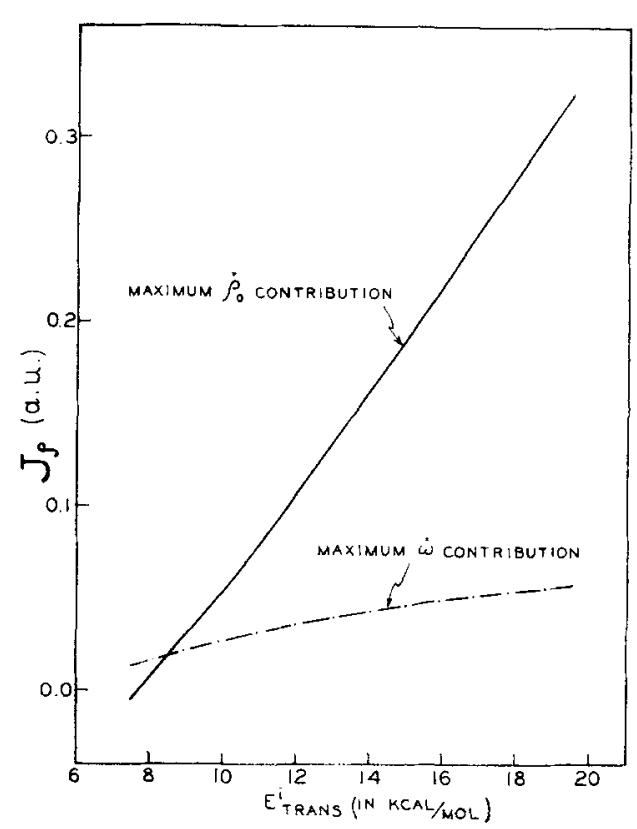

FIG. 14. Maximum contributions from $\dot{\rho}_{0}$ and $\dot{\omega}$ terms to the final action $J_{\rho}$ (maximum over-all vibrational phases) as a function of initial relative translational energies.

some instances, reflection and hence nonreactivity occurs after the system has passed through the saddlepoint region. This reflection, involving as it does a recrossing of line $L$, leads to a decrease in validity of activated complex theory at high energies.

To explore the last and other points a number of trajectories were plotted in Figs. 18 and 19. For these collisions, which have more than sufficient translational energy to overcome the adiabatic threshold, the nonreactivity is seen to occur as a result of reflection after the system has crossed the activated complex line $L$ $(s=0)$. Several other observations from these figures may also be made:

(1) There are two trajectories (No. 1 and one near No. 3, hereinafter referred to as No. 3) which are symmetric about the activated complex line $L$, and for these, of course, $\dot{\rho}=0$ and $\dot{\rho}-\dot{\rho}_{0}=0$ on line $L$. Thus,

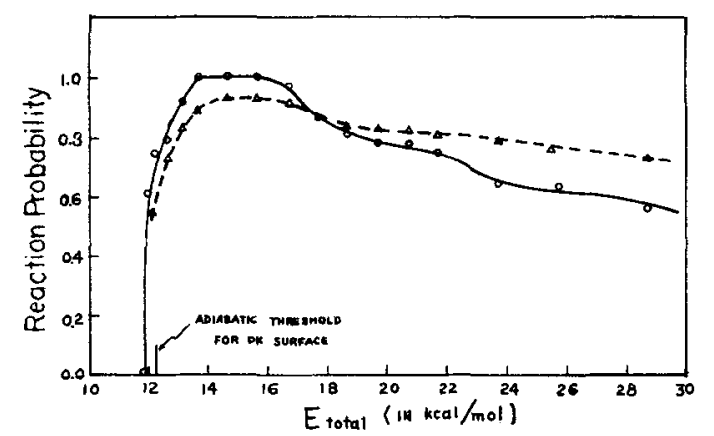

HIG. 15. Reaction probability for linear collision as a function of total energy: - exact classical calculation; - - -, approximate calculation based on Eqs. (23) and (14). 
such trajectories cross line $L$ at a turning point of vibrational $\rho$ motion. These points lie to the left of the saddle point, as expected from the internal centrifugal force term $\alpha / \eta^{2}$. According to Figs. 18 and 19 for the exact trajectories, $E_{\mathrm{vib}}$ at line $L$ and $J_{\rho}$ at line $L$ should be less for the symmetric trajectory 1 than for 3 since the vibrational potential energy is smaller in the former case and both have zero vibrational kinetic energy. This observation is also in agreement with the results for the approximate $J_{\rho}$ 's based on Eq. (14) and given in Figs. 16 and 17, where trajectory 1 has a smaller $J_{\rho}$ at $s=0$ than does trajectory 3.

(2) All the reactive trajectories were found to cross line $L$ in an interval $(a, b)$, which is roughly bisected by the reaction coordinate. Thus, the ensemble of systems crossing $L$ are more "centered" on the reaction

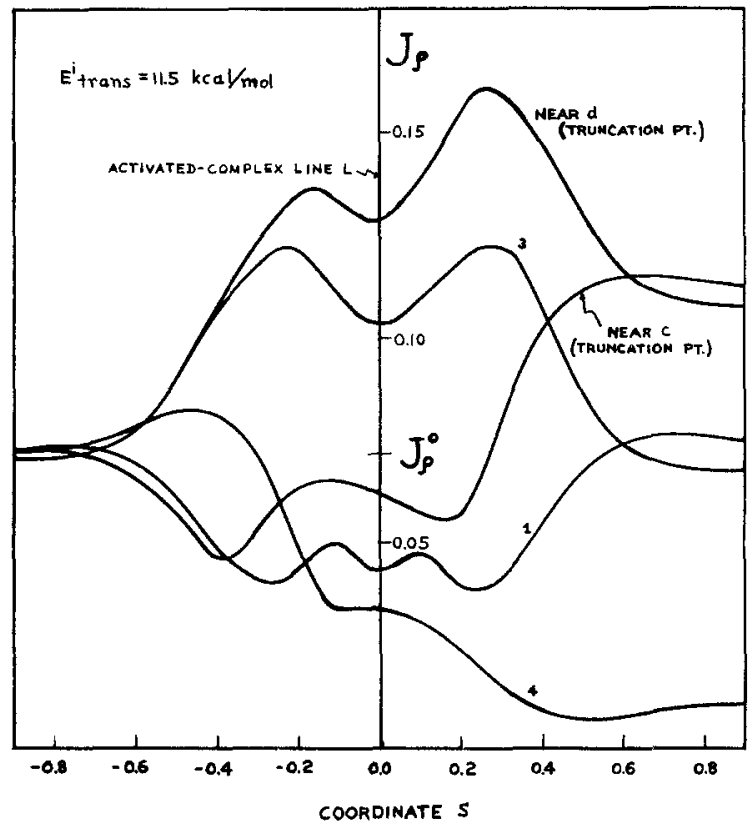

FIG. 16. Time evolution of the vibrational action $J_{\rho}(s)$ in a linear collision for $E_{\mathrm{trang}}{ }^{i}=11.5 \mathrm{kcal} / \mathrm{mol}$. The corresponding initial vibrational phases are indicated in Fig. 9.

coordinate than on the curve of steepest descent (the "reaction path"), curve $C$ in Fig. 1. Thereby, as predicted in Ref. 1, the use of a reaction coordinate which includes the dynamical curvilinear effect, as in the present case, provides a better zeroth-order separability between the vibrational and translational motions of the system than the use of the "reaction path" alone.

An effort was made to see if even better agreement of approximate and exact calculations in Figs. (6)-(11) could be made by integrating the approximate Eqs. (13) exactly ${ }^{29}$ Agreement was somewhat better in some regions, somewhat worse in others, and that more hybrid procedure was not explored further.

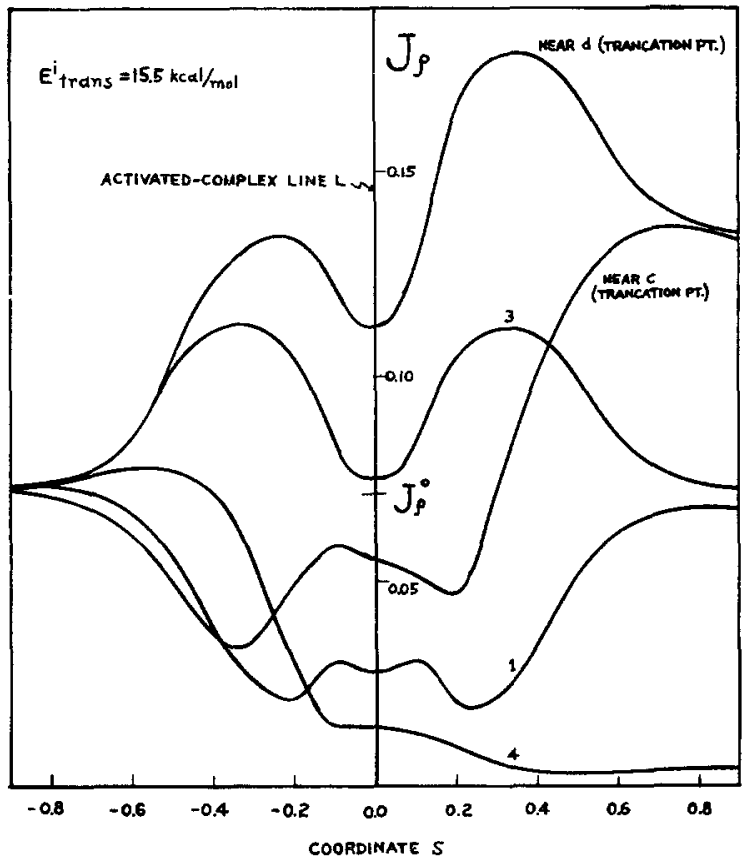

Frg. 17. Time evolution of the vibrational action $J_{\rho}(s)$ in a linear collision for $E_{\text {trans }}{ }^{i}=15.5 \mathrm{kcal} / \mathrm{mol}$. The corresponding initial vibrational phases are indicated in Fig. 11.

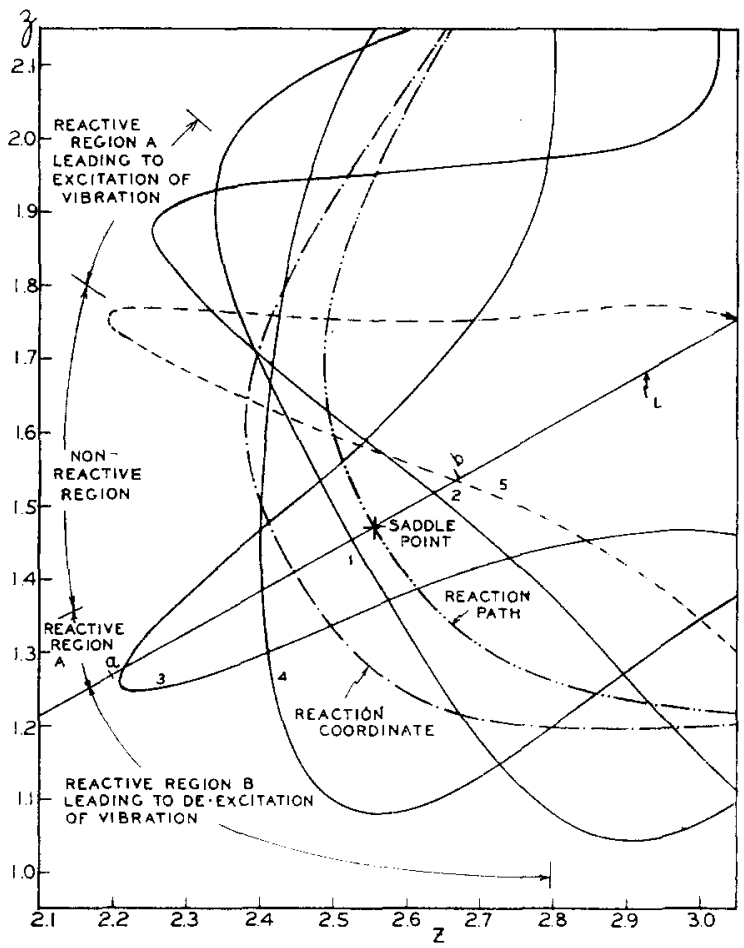

FIg. 18. Plot of classical trajectories on the potential energy surface near the saddle-point region, for the five vibrational phases indicated in Fig. 9: - , reactive trajectory; ..., nonreactive trajectory. $\left(E_{\operatorname{tran}} i=11.5 \mathrm{kcal} / \mathrm{mol}\right.$.) Curve $\mathcal{C}(\ldots-\ldots)$ and the reaction coordinate (--) are also plotted. 


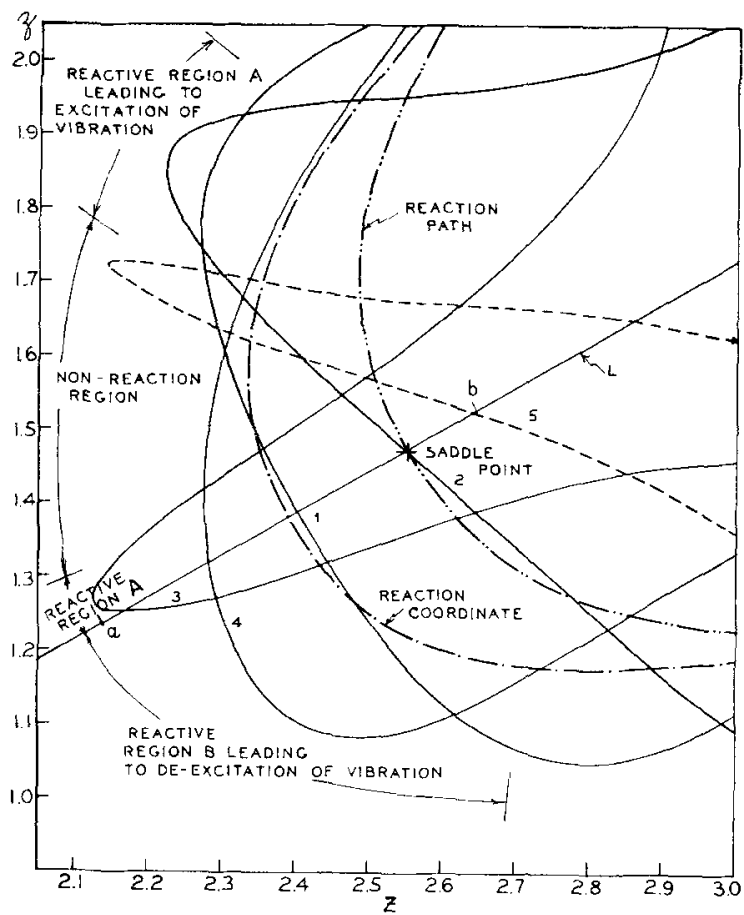

Fic. 19. Plot of classical trajectories on the potential energy surface near the saddle-point region, for the five vibrational phases indicated in Fig. 11: - , reactive trajectory; - ., nonreactive trajectory. $\left(E_{\mathrm{trans}}{ }^{i}=15.5 \mathrm{kcal} / \mathrm{mol}\right)$. Curve $C(-\cdots-)$ and the reaction coordinate $(-\cdot-)$ are also plotted.

\section{CONCLUSIONS}

Application of the Hamilton-Jacobi equation in the near-adiabatic approximation, and of local action-angle variables, was made to the linear reactive $\mathrm{H}+\mathrm{H}_{2}$ system. Good agreement was found between the exact and approximate calculation for the absolute value of the vibrationally phase-averaged vibrational energy change $\left\langle\Delta E_{\mathrm{vib}}\right\rangle$ accompanying reaction, in the region investigated ( $E_{\text {trans }}{ }^{i}$ from 7 to $20 \mathrm{kcal} / \mathrm{mol}$ ). (At the usual reaction temperatures of $500-1200^{\circ} \mathrm{C}$, the $E_{\text {trans }}{ }^{i}$ contributing significantly to the reaction collisions on this surface would be in the neighborhood of $8-10.5 \mathrm{kcal} /$ mol.) Good agreement for the phase-dependent $\Delta E_{\mathrm{vib}}$ was also found for the data in Figs. 6-11. In effect, the curvilinear $\left[\dot{\rho}_{0}\right]$ term in (14) was treated as a perturbation to second or higher order, while the $\dot{\omega}$ term was treated as a perturbation to first order, a method which later proved to be consistent with the findings in Figs. 13 and 14. Again, the phase-averaged vibrational energy change, $\left\langle\Delta E_{\mathrm{vib}}\right\rangle$ in Fig. 12, was small for $E_{\operatorname{trans}}{ }^{i}$ in the range appropriate to the usual reaction temperatures of $500-1200^{\circ} \mathrm{K}$.

The centering of the reactive trajectories on the reaction coordinate defined by $\mathrm{Eq}$. (7), as those trajectories crossed line $L$, lends further support to the concepts employed in (7)-(10). In the corresponding quantum mechanical treatment, a more truncated vibrational basis set can be used at each $s$ if that set is centered upon a mean reaction coordinate curve [de- fined by some $\left.\rho_{0}(s)\right]$ as compared with centering it on the curve $C$ in Fig. 1.

On the basis of these results one can expect that the corresponding quantum treatment will attain similar good agreement, at least when tunneling effects in the $s$ motion can, as in the present classical case, be neglected. Tunneling can, incidentally, lead to one difficulty in the case of the present coordinates: It causes a "negative" internal centrifugal force ${ }^{1 a}$ since $\alpha(s)$ in Eq. (6) is negative, and can thus cause the system to come near the singularity at $1+\kappa \rho=0$ if curve $C$ is selected as in Fig. 1 . In such cases it may be necessary to use as curve $C$ a curve appreciably displaced from the steepest descent curve in Fig. 1, or to solve the problem in the presence of the singularity.

The findings of a small $\left\langle\Delta E_{\mathrm{vib}}\right\rangle$ at low energies in Fig. 12 indicate that the reaction is statistically adiabatic $^{30}$ at the usual reaction energies, even though, as Figs. 6-11 show, it is not exactly adiabatic. ${ }^{31}$ Thus, upon energy averaging at a particular temperature, activated complex theory should be valid for describing the reaction rate in the classical system described in this paper. Deviations could nevertheless occur at low translational energies and hence at low temperatures because of the nonadiabatic leak, at least in the present case. Deviations would also occur at very high energies, and, thereby, at extremely high temperatures, due to the recrossing of the activated complex line $L$ discussed in the preceding section.

This concept of adiabaticity has provided a zerothorder basis for separating the motions perpendicular to the reaction coordinate from that parallel to that coordinate. This separation is the more valid the more slowly the molecular properties (such as the rate of change of vibrational frequency and the rate of change of tangent vector of the reaction curve) vary along that curve. Natural collision coordinates are particularly suited to such system, and hence to understanding activated complex theory. They should become less useful when the molecular properties vary rapidly along the curve $C$, e.g., when the curvature $\kappa(s)$ becomes very large. Such reactions are strongly nonadiabatic and are undoubtedly better treated by an impulse type of approximation.

* Acknowledgement is made to the donors of the Petroleum Research Fund, administrated by the American Chemical Society, for partial support of this research. This research was also supported by a grant from the National Science Foundation at the University of Illinois.

1 (a) R. A. Marcus, J. Chem. Phys. 45, 4493 (1966), Paper I; (b) 45, 4500 (1966), Paper II; (c) 49, 2610 (1968), Paper III; (d) Discussions Faraday Soc. 44, 7 (1967), and discussion remarks.

${ }^{2}$ R. A. Marcus, J. Chem. Phys. 49, 2617 (1968), Paper IV. In Eq. (93) the $\left(2 J_{\rho}^{0}\right)^{1 / 2}$ should read $2\left(J_{p}^{0}\right)^{1 / 2}$.

(a) D. Russell and J. C. Light, J. Chem. I'hys. 51, 1720 (1969); (b) C. C. Rankin and J. C. Light, ibid. 51, 1701 (1969).

${ }^{4}$ R. E. Wyatt, J. Chem. Phys. 51, 3489 (1969).

5 J. N. L. Connor and M. S. Child, Mol. Phys. 18, 653 (1970). 6 See, e.g., M. Born, The Mechanics of the Atom (Ungar, New York, 1960)

7 (a) A. O. Cohen and R. A. Marcus, J. Chem. Phys. 49, 4509 (1968); (b) 52, 3140 (1970). 
${ }^{8}$ M. Attermeyer and R. A. Marcus, J. Chem. Phys. 52, 393 (1970)

${ }^{9}$ Action-angle variables have also been applied to the calculation of transition probabilities by W. H. Miller (private communication) and by the author.

${ }^{10}$ F. T. Wall, L. A. Hiller, Jr., and J. Mazur, J. Chem. Phys. 29, 255 (1958); 35, 1284 (1961); F. T. Wall and R. N. Porter, ibid. 39, 3112 (1963).

${ }_{11}$ M. Karplus, R. N. Porter, and R. D. Sharma, J. Chem. Phys. 43, 3259 (1965); 40, 2033 (1964).

12 (a) R. A. Marcus, J. Chem. Phys. 46, 959 (1967); (b) Ref. 1(b).

${ }^{13}$ E. M. Mortensen, J. Chem. Phys. 49, 3526 (1968).

14 E. M. Mortensen and K. S. Pitzer, Chem. Soc. (London), Spec. Publ. 16, 57 (1962) ; E. M. Mortensen, J. Chem. Phys. 48, 4029 (1968)

${ }^{15}$ D. Micha, Arkiv Fysik 30, 425, 437 (1965); J. Chem. Phys. $50,722(1969)$.

${ }_{16}$ (a) M. Karplus and K. T. Tang, Discussions Faraday Soc. 44, 56 (1967); (b) K. T. Tang and M. Karplus, J. Chem. Phys. 49,1676 (1968).

${ }^{17} \mathrm{H}$. C. Corben and P. Stehle, Classical Mechanics (Wiley, New York, 1960).

${ }^{18} \alpha$ is the $\alpha_{k}$ of Ref. 2. We have, in effect, utilized Eqs. (11) $(16),(81)$ and (82) of Ref. 2 to obtain Eq. (6), without necessarily employing the local-harmonic approximation used in (81). The $\epsilon$ in Eq. (81) is the present $E_{\mathrm{vib}}(s)$.

${ }^{19}$ We have chosen an arbitrary constant, $t_{0}$, in (93) and (94) to be zero.

${ }^{20}$ See, for example, references cited in I. Shavitt, R. M. Stevens, F. L. Minn, and M. Karplus, J. Chem. Phys. 48, 2700 (1968).

${ }^{21}$ R. N. Porter and M. Karplus, J. Chem. Phys. 40, 1105 (1964).
${ }^{22}$ The equations of motion in Cartesian coordinates ${ }^{9,10}$ are $\dot{z}=p_{z} / \mu, \dot{p}_{z}=-\partial V / \partial z, \dot{Z}=p_{z} / \mu, \dot{p}_{z}=-\partial V / \partial Z$, where $V$ is the potential energy. At end of the calculation $z$ and $Z$ are converted to Cartesian coordinates appropriate to products.

${ }^{23}$ The step size of the integration, $\Delta t$, was $0.02 \times 10^{-14} \mathrm{sec}$, which was approximately $1 / 25$ of the vibrational period of $\mathbf{H}_{2}$ molecule. Computing time on the IBM 7094 for one initial relative translational energy with 36 vibrational phases was approximately $10 \mathrm{~min}$ with a step size of $0.02 \times 10^{-14} \mathrm{sec}$; the exact value depended upon the initial relative velocity.

${ }^{24}$ Ref. 1 (a), Footnote 16.

${ }^{25}$ Computing for a single translational energy and an arbitrary number of vibrational phases was $2 \mathrm{~min}$.

${ }^{26}$ The rigorous solution for $s$ in (23) is straightforward, but the needed integrals in (14) were not printed out.

${ }^{27}$ The more rigorous solution of $(23)^{26}$ would have lowered the transmissions at high $E_{\text {trans }}{ }^{i}$, and so perhaps have improved the agreement in Fig. 15.

${ }^{28}$ Actually the results in these figures are obtained from the integration of the equations for the $\phi_{1}$ and $\phi_{2}$ functions in Ref. 29.

${ }^{29}$ For this purpose real combinations of the $a$ and $a^{*}$ were introduced, defined by $\phi_{1}=J_{\rho}^{1 / 2} \cos 2 \pi w_{\rho}, \phi_{2}=J_{\rho}^{1 / 2} \sin 2 \pi w_{\rho}$, and the resulting equations for $\phi_{1}$ and $\phi_{2}$ were integrated numerically.

${ }^{30}$ R. A. Marcus, J. Chem. Phys. 49, 2617 (1968); Ref. 12(b).

${ }^{31}$ For the relation of adiabatic theory to activated complex theory see, for example, M. A. Eliason and J. O. Hirschfelder, J. Chem. Phys. 30, 1426 (1959), and other references in R. A. Marcus, ibid. 45, 4493 (1966), and Appendix III of Ref. 11(a). Incidentally, an adiabaticity or statistical adiabaticity from reactants' region to activated complex region suffices to yield activated complex theory, provided no subsequent reflection of the system occurs during its motion from activated complex region to products region.

\title{
Pseudopotential Approaches to Localized Orbitals for Polyatomic Systems
}

\author{
Herbert Schlosser \\ Department of Physics, Cleveland State University, Cleveland, Ohio 44115
}

(Received 17 July 1970)

\begin{abstract}
We present a method for constructing optimized pseudopotentials for general polyatomic systems which yield the "smoothest" orbitals localized in particular regions of space. " Smoothest" orbitals are those for which the expectation value of the kinetic energy operator is a minimum.) We specifically consider the general case in which one can have several occupied orbitals localized in a particular region, rather than a single orbital. Recently, Anderson presented a pseudopotential formalism for constructing localized orbitals for a single isolated band in a polyatomic system. We have extended his formalism to the general multiorbital case, and show that for a specific choice of pseudopotential the Anderson equation yields optimal orbitals. We also show that the Hückel form of the Anderson pseudopotential equation yields identical eigenvalues (neglecting terms of order $S^{2}$ ) to the optimal form, and orbitals differing from optimal orbitals by terms linear in $S$. The correction terms to the Hückel orbitals are obtained by first-order perturbation theory. Finally we examine the Adams-Gilbert equation, which is valid only for systems representable by a single Slater determinant, and indicate how one may solve this equation for the localized orbitals using perturbation theory with Anderson orbitals as the basis set.
\end{abstract}

\section{INTRODUCTION}

It has been pointed out a number of times in the past $^{1-4}$ that functions which are spacially localized are extremely useful in describing the one electron orbitals in molecular systems and in a number of solid state problems. Adams ${ }^{2}$ and Gilbert ${ }^{3}$ independently showed how one may obtain localized orbitals for polyatomic systems by appropriate transformation of Hartree-Fock (HF) orbitals for the system. However, their results are limited to closed-shell situations.
Recently Anderson presented a self-consistent pseudopotential method for constructing extremely localized orbitals for a single isolated band in solids or molecules, ${ }^{4}$ and applied this formalism to derive the Hückel approximation of molecular theory in a rigorous manner. ${ }^{5}$ Subsequently, Kunz ${ }^{6}$ presented a method for simplifying the solution of the Adams-Gilbert (AG) equation by means of expansions in powers of the interatomic overlap and iterative solution of the resultant equation. In a previous paper $^{7}$ we presented an alternative scheme for simplification of the $A G$ equation by 